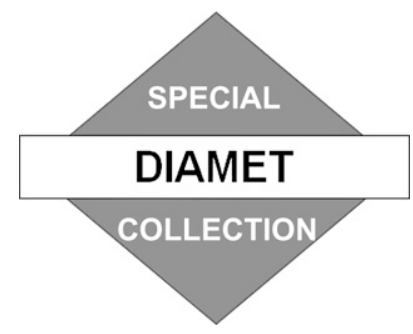

\title{
¿Does the Representation of Flow Structure and Turbulence at a Cold Front Converge on Multiscale Observations with Model Resolution? $\mathscr{O}$
}

\author{
Ben HARVEY \\ National Centre for Atmospheric Science, University of Reading, Reading, United Kingdom \\ JOHN METHVEN \\ Department of Meteorology, University of Reading, Reading, United Kingdom \\ Chloe Eagle and Humphrey Lean \\ MetOffice@Reading, University of Reading, Reading, United Kingdom
}

(Manuscript received 20 December 2016, in final form 18 August 2017)

\begin{abstract}
In situ aircraft observations are used to interrogate the ability of a numerical weather prediction model to represent flow structure and turbulence at a narrow cold front. Simulations are performed at a range of nested resolutions with grid spacings of $12 \mathrm{~km}$ down to $100 \mathrm{~m}$, and the convergence with resolution is investigated. The observations include the novel feature of a low-altitude circuit around the front that is closed in the frame of reference of the front, thus allowing the direct evaluation of area-average vorticity and divergence values from circuit integrals. As such, the observational strategy enables a comparison of flow structures over a broad range of spatial scales, from the size of the circuit itself $(\approx 100 \mathrm{~km})$ to small-scale turbulent fluctuations $(\approx 10 \mathrm{~m})$. It is found that many aspects of the resolved flow converge successfully toward the observations with resolution if sampling uncertainty is accounted for, including the area-average vorticity and divergence measures and the narrowest observed cross-frontal width. In addition, there is a gradual handover from parameterized to resolved turbulent fluxes of moisture and momentum as motions in the convective boundary layer behind the front become partially resolved in the highest-resolution simulations. In contrast, the parameterized turbulent fluxes associated with subgrid-scale shear-driven turbulence ahead of the front do not converge on the observations. The structure of frontal rainbands associated with a shear instability along the front also does not converge with resolution, indicating that the mechanism of the frontal instability may not be well represented in the simulations.
\end{abstract}

\section{Introduction}

Atmospheric frontal systems are associated with numerous high-impact weather phenomena. The majority of extreme precipitation events in midlatitudes are

Denotes content that is immediately available upon publication as open access.

Supplemental information related to this paper is available at the Journals Online website: https://doi.org/10.1175/MWR-D-16-0479.s1.

Corresponding author: Ben Harvey, b.j.harvey@reading.ac.uk associated with fronts (Catto and Pfahl 2013), and intense wind gusts, including tornadoes, commonly occur near frontal rainbands (Clark and Parker 2014). Despite being embedded within large-scale weather systems, often stretching over thousands of kilometers, the narrow cross-frontal scale of frontal systems coupled with

Publisher's Note: This article was revised on 16 September 2017 to include the CCBY license that was missing when originally published.

This article is licensed under a Creative Commons Attribution 4.0 license (http://creativecommons. org/licenses/by/4.0/). 
often intense meso- and convective-scale circulations mean they continue to provide a challenge for numerical weather prediction (NWP) models.

In the idealized, frictionless semigeostrophic limit, frontogenetic motions acting on a baroclinic zone cause the collapse of the cross-frontal scale to zero in a finite time (Hoskins and Bretherton 1972). While in reality this collapse is halted by other processes neglected in that model, albeit not fully understood ones, observed frontal zones are often found to be narrower than can be resolved by current NWP models. Therefore, the modeled frontal widths are typically set artificially by (implicit or explicit) numerical diffusive effects, rather than by resolved physical motions. The degree to which the prediction of associated high-impact weather is affected by this limitation is not understood.

Operational local-area forecast models are now approaching convection-permitting grid spacings of $O(1) \mathrm{km}$ (Clark et al. 2016). In such models deep tropospheric convection is at least partially resolved (Lean et al. 2008) and fronts with cross-frontal scales of several kilometers should be partially represented. However, boundary layer turbulence, which is known to strongly influence frontal structure and the representation of associated high-impact surface weather, remains poorly resolved at these resolutions (e.g., Williams 1974; Sinclair and Keyser 2015). Development is also underway at several forecasting centers on experimental local-area NWP models with subkilometer resolutions, down to $O(100) \mathrm{m}$ grid spacings. In this case, shallow boundary layer convective motions will also be partially resolved, resulting in a reduced need for the parameterization of nonlocal boundary layer mixing. Such models have been shown to have an improved representation of summertime U.K. convection (Stein et al. 2015; Hanley et al. 2015), cold pooling in valleys (Vosper et al. 2013), the formation of marine stratocumulus (Boutle et al. 2014), and the formation of tornado-like structures in free-running simulations over the U.S. Great Plains (Hanley et al. 2016). However, the validation of such models for fast-moving dynamical features such as fronts is problematic because of the limitations on domain size and a lack of suitable observations.

In this study, the representation of a mature ana-type cold front in a high-resolution NWP model is interrogated across a range of model resolutions with grid spacings of $12 \mathrm{~km}$ down to $100 \mathrm{~m}$. A key feature is the use of novel in situ aircraft observations that include a closed circuit around the front within the boundary layer. This observational strategy enables the direct evaluation of area-average vorticity and convergence at the front via circuit integral techniques. Since the front is fast moving, the aircraft circuit is not closed in the
Earth-relative frame of reference but rather designed with the aim of being approximately closed in the frame of reference moving with the front. Together with observations of local wind speeds and vertical turbulent fluxes, the circuit integrals provide a detailed evaluation of the convergence of the model with resolution against reality at a range of spatial scales. A series of nested numerical simulations is employed, spanning the range from a traditional NWP model (12-km grid spacing) in which both tropospheric and boundary layer convective mixing are performed by parameterization schemes, through convection-permitting resolutions in which the convection scheme is switched off (2.2- and $1.5-\mathrm{km}$ grid spacings) and down to subkilometer resolution models (500-, 200-, and 100-m grid spacings) in which both the convection scheme and the nonlocal boundary layer mixing scheme are switched off.

Ana-type cold fronts typically exhibit sharp frontal transition zones in the boundary layer, accompanied by strong updrafts and a narrow band of relatively heavy precipitation called a narrow cold-frontal rainband (NCFR) (e.g., Browning and Harrold 1970). Such rainbands are often observed to break up into line segments separated by gaps of weaker or no precipitation (James and Browning 1979; Hobbs and Biswas 1979). The mechanism of the break up is usually attributed to a horizontal shear instability, whereby the band of strong horizontal shear along the frontal transition zone is unstable and the resulting motion acts to wrap the strip of vorticity into a series of coherent vortices (Hobbs and Persson 1982; Kawashima 2011). In terms of surface impacts, the most intense precipitation along the front falls between the vortices, on narrow filaments of strong shear and temperature gradient, and tornadic structures, when they occur in the United Kingdom, typically also occur in the braids joining such vortices (Clark and Parker 2014; Mulder and Schultz 2015).

The presence of such rainband segments in the case studied here provides both opportunities and complications. By good fortune, the aircraft circuit crossed both a narrow filament of strong shear on one frontal transect and a coherent vortex structure on the other, thus enabling a comparison of both features within the model simulations. However, since the rainband segments are associated with strong alongfront inhomogeneities, care is needed with the area-average vorticity and divergence observations to ensure they are representative of the front as a whole.

The paper is organized as follows. In section 2 the flight track and instrumentation are summarized. The numerical model is also described, including details of the dynamical core and physics parameterizations. In section 3 a synoptic overview of the case is presented, 


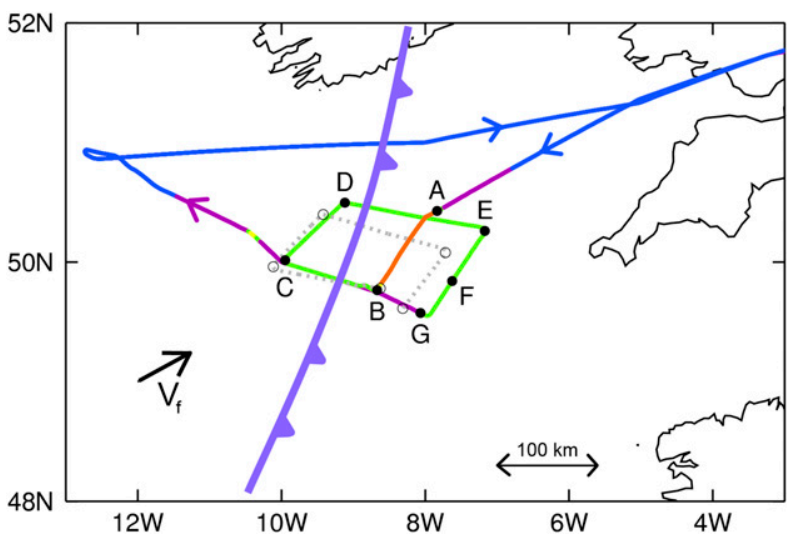

FIG. 1. The flight track and the approximate position of the front at 1500 UTC. Color indicates altitude, increasing from orange for altitudes below $200 \mathrm{~m}$ to green, purple, and blue at levels above $200 \mathrm{~m}, 400 \mathrm{~m}$, and $4 \mathrm{~km}$, respectively. The displacement of the front over $1 \mathrm{~h}$ is indicated by the black arrow. The flight track shifted to a frame relative to the front at $t_{\text {ref }}=1500$ UTC (see section 2c; gray dotted line). Locations $\mathrm{A}-\mathrm{G}$ on the flight track (black dots), and the corresponding points $\mathrm{B}-\mathrm{F}$ on the shifted flight track (open circles). The timing of all the labeled points is shown in Fig. 4.

highlighting the presence of segmented rainbands along the front. In section 4 the convergence of the resolved flow with increased model resolution is addressed, and in section 5 the representation of vertical turbulent fluxes in the boundary layer is considered. In section 6 the structure of the frontal rainband segments in the model is discussed, and the conclusions and further discussion are presented in section 7 .

\section{Data and methods}

\section{a. Flight track and instrumentation}

The case studied here is from 24 November 2009, during which an area of deep low pressure developed to the west of Scotland and the associated trailing cold front advanced from the west toward the United Kingdom and France. During the afternoon of that day, the Facility for Airborne Atmospheric Measurements (FAAM) BAe146 aircraft undertook a research flight, as part of the THORPEX North Atlantic Waveguide and Downstream Impact Experiment (T-NAWDEX) pilot campaign (Knippertz et al. 2010; Vaughan et al. 2015), over the southwest approaches of the United Kingdom to examine the structure of the front. There were two aims: to take detailed in situ measurements of the frontal structure at low altitude $(300 \mathrm{~m})$, including the frontal circuit and measurements of turbulent fluxes; and to observe the vertical structure of the warm conveyor belt, including measurements of the associated moisture transport. The warm conveyor belt observations are discussed in Martínez-Alvarado et al. (2014); the focus of the present study is on the near-surface frontal structure and its representation in the numerical simulations.

Figure 1 depicts the route of the flight track together with the approximate position of the front at 1500 UTC 24 November 2009. The FAAM BAe-146 aircraft took off from Cranfield Airport, England, at 1311 UTC and flew southwestward at cruising altitude, reaching the vicinity of the front at 1439 UTC (point A). Two low-level maneuvers were then performed: a 100-km alongfront leg (labeled AB) slightly ahead of the front at 40-m altitude (1439-1456 UTC) and a front-crossing rectangular circuit (labeled BCDEG) at 300-m altitude (1456-1604 UTC) of size $80 \mathrm{~km} \times 140 \mathrm{~km}$.

Leg AB provides measurements of surface-layer turbulent fluxes in storm-force winds and estimated peakto-trough ocean wave heights of $6-12 \mathrm{~m}$. The circuit BCDEG was designed to be closed in a frame of reference moving with features on the front, but in practice the circuit was found to be best closed by a point between $\mathrm{E}$ and G, labeled F in Fig. 1 (see section 3b). Subsequently, the aircraft turned to cross the front again before ascending through the cold-sector boundary layer (1635-1705 UTC) and finally crossing the front at high altitude to produce a vertical cross section of the front from dropsonde data (1705-1742 UTC; see Fig. 4 of MartínezAlvarado et al. 2014). An air-relative speed of $200 \mathrm{kt}$ $\left(\approx 100 \mathrm{~m} \mathrm{~s}^{-1}\right.$ ) was maintained throughout the flight.

Full details of the instrumentation carried by the aircraft are given by Renfrew et al. (2008), Petersen and Renfrew (2009) and Vaughan et al. (2015), and the in situ observations used here are available from FAAM (2014). The key to this study is a Rosemount 102BL temperature sensor and the FAAM five-port wind and turbulence probe, both of which report measurements at $32 \mathrm{~Hz}$ (approximately 3-m spacing at science speed) with precision of $\pm 0.3 \mathrm{~K}$ and $\pm 0.25 \mathrm{~m} \mathrm{~s}^{-1}$, respectively. Use is also made of specific humidity measurements from a Lyman-alpha hygrometer that reports measurements at $64 \mathrm{~Hz}$ with an accuracy of $\pm 0.15 \mathrm{~g} \mathrm{~kg}^{-1}$, although these data are resampled to $32 \mathrm{~Hz}$ to match the other variables. Turbulent fluxes are calculated following the methodology of Cook and Renfrew (2015), as follows. Each low-level leg of the flight track is split into straight and level runs of 2-min duration $(\approx 12 \mathrm{~km})$. Run-average vertical fluxes of sensible heat, latent heat, and momentum are then calculated as

$$
\begin{aligned}
\mathrm{SH} & =\bar{\rho} c_{p} \overline{w^{\prime} \theta^{\prime}}, \\
\mathrm{LH} & =\bar{\rho} L_{v^{w^{\prime} q^{\prime}},} \\
\tau & =\bar{\rho} \sqrt{{\overline{u^{\prime} w^{\prime}}}^{2}+{\overline{v^{\prime} w^{\prime}}}^{2}},
\end{aligned}
$$


TABLE 1. Model configurations used in this study; Smag = Smagorinsky.

\begin{tabular}{|c|c|c|c|c|c|c|c|}
\hline Resolution & $\begin{array}{l}\text { Approximate domain } \\
\text { size }(\mathrm{km} \times \mathrm{km})\end{array}$ & $\begin{array}{l}\text { Time } \\
\text { step }\end{array}$ & $\begin{array}{l}\text { Convection } \\
\text { scheme }\end{array}$ & $\begin{array}{l}\text { Boundary layer } \\
\text { scheme }\end{array}$ & $\begin{array}{l}\text { Subgrid mixing } \\
\text { scheme }\end{array}$ & $\begin{array}{l}\text { Initialization } \\
\text { time }\end{array}$ & $\begin{array}{l}\text { Boundary updating } \\
\text { frequency }\end{array}$ \\
\hline $40 \mathrm{~km}, \mathrm{~L} 70$ & Global & $12 \mathrm{~min}$ & On & On & Off & 0600 UTC 23 Nov & - \\
\hline $12 \mathrm{~km}, \mathrm{~L} 38$ & $10000 \times 6100$ & $5 \mathrm{~min}$ & On & On & Off & 0600 UTC 23 Nov & $3 \mathrm{~h}$ \\
\hline $2.2 \mathrm{~km}, \mathrm{~L} 70$ & $3100 \times 2700$ & $75 \mathrm{~s}$ & Off & On & 2D Smag & 1200 UTC 23 Nov & $30 \mathrm{~min}$ \\
\hline $1.5 \mathrm{~km}, \mathrm{~L} 70$ & $1600 \times 1500$ & $50 \mathrm{~s}$ & Off & On & 2D Smag & 1500 UTC 23 Nov & $30 \mathrm{~min}$ \\
\hline 500 m, L140 & $850 \times 600$ & $10 \mathrm{~s}$ & Off & Off & 3D Smag & 1800 UTC 23 Nov & $15 \mathrm{~min}$ \\
\hline 200 m, L140 & $320 \times 320$ & $6 s$ & Off & Off & 3D Smag & 1200 UTC 24 Nov & $15 \mathrm{~min}$ \\
\hline $100 \mathrm{~m}, \mathrm{~L} 140$ & $150 \times 150$ & $3 \mathrm{~s}$ & Off & Off & 3D Smag & 1200 UTC 24 Nov & $15 \mathrm{~min}$ \\
\hline
\end{tabular}

respectively, where $u^{\prime}, v^{\prime}, w^{\prime}, \theta^{\prime}$, and $q^{\prime}$ are perturbations of the wind components, potential temperature, and humidity for the run, detrended for each run; and the overbars denote run-average values. In addition, $\bar{\rho}$ is the run-average air density, $c_{p}=1004 \mathrm{~J} \mathrm{~kg}^{-1} \mathrm{~K}^{-1}$ is the specific heat capacity for dry air, and $L_{v}=$ $2.5 \times 10^{6} \mathrm{~J} \mathrm{~kg}^{-1} \mathrm{~K}^{-1}$ is the latent heat of vaporization. Cook and Renfrew (2015) apply a surface-layer correction to $\tau$ in order to obtain an estimate of the value at the surface; this correction is not applied here, since the values are compared directly with the model output at the aircraft altitude.

\section{b. Numerical model}

The simulations presented here are performed with the Met Office Unified Model (UM), version 8.4, which employs a nonhydrostatic, deep-atmosphere dynamical core with a semi-Lagrangian time-stepping scheme (Davies et al. 2005). Six limited-area simulations are performed with resolutions ranging from $12-\mathrm{km}$ grid spacing with 38 levels to $100-\mathrm{m}$ grid spacing with 140 levels, as described in Table 1 . The $12-\mathrm{km}$ model takes its initial and boundary conditions from a global simulation with $40-\mathrm{km}$ grid spacing, and each subsequent resolution is one-way nested from the previous. The model domains are shown in Fig. 2. The presence of extreme strong winds and the fast propagation speed of the front itself provide a substantial computational challenge. To allow time for the spinup of small-scale features as the front enters each subdomain, the domains are made as large as practically possible. In addition, the nesting of each submodel, which is achieved by passing boundary conditions from each parent model to each submodel at a predetermined updating frequency (see Table 1), is made as frequent as practically possible.

The global and 12-km limited-area models employ the following parameterization schemes: the radiation scheme of Edwards and Slingo (1996), the surface-layer scheme of Best et al. (2011), the mixed-phase cloud microphysics scheme of Wilson and Ballard (1999), the nonlocal boundary layer scheme of Lock et al. (2000), and a convection scheme based on Gregory and Rowntree (1990). At resolutions of $2.2 \mathrm{~km}$ and below where the convection scheme is switched off, an additional local subgrid turbulent-mixing scheme is used (Halliwell 2007). Subgrid turbulent mixing is an essential component of NWP models with grid spacings of $0.1-1.5 \mathrm{~km}$, since at these resolutions the boundary layer inertial subrange is at best only partially resolved. The simulations presented here use a Smagorinsky-Lillytype scheme that acts either just in the horizontal with vertical mixing provided by the nonlocal boundary layer scheme (2.2- and $1.5-\mathrm{km}$ simulations) or in all three spatial dimensions with the boundary layer scheme switched off (500-, 200-, and 100-m simulations). In all cases the subgrid mixing length is set to 0.2 times the horizontal grid spacing. These configurations of the Met Office Unified Model were developed during a number of previous studies, including Vosper et al. (2013), Hanley et al. (2015), and Stein et al. (2015).

\section{c. Methodology for model-observations comparison}

To perform circuit integrals in the front-relative frame of reference, the following change of coordinates is

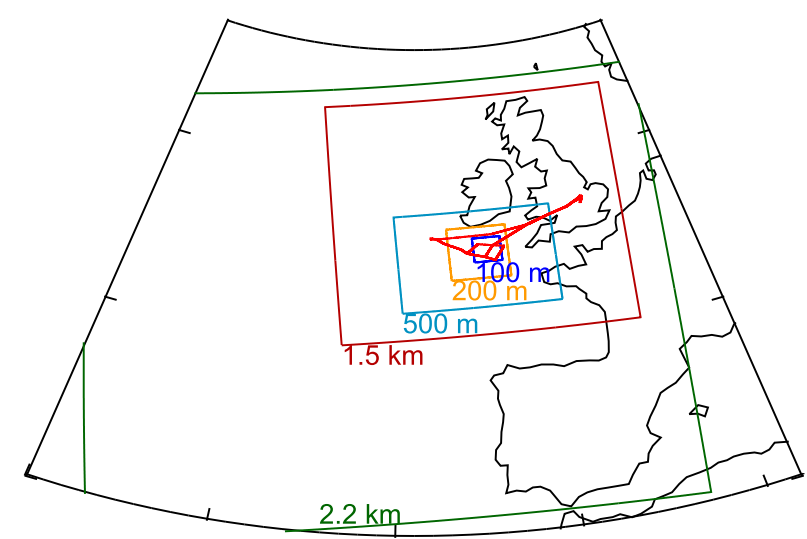

FIG. 2. The model domains used for the $2.2-\mathrm{km}, 1.5-\mathrm{km}, 500-\mathrm{m}$, 200-m, and 100-m simulations in this study. The domain of the $12-\mathrm{km}$ simulation is much larger than the region shown, extending from $90^{\circ} \mathrm{W}$ to $75^{\circ} \mathrm{E}$ at the latitude of the flight track and $25^{\circ}-80^{\circ} \mathrm{N}$ at the longitude of the flight track. The flight track is indicated (red line). 
performed to the flight track. First, a reference time $t_{\mathrm{ref}}$ is chosen for which model output is available (for instance, $t_{\text {ref }}=1500$ UTC). Then, each location along the flight track $\mathbf{x}_{\text {obs }}(t)$ is shifted to its position relative to the front at $t_{\text {ref }}$ according to

$$
\mathbf{x}_{\mathrm{rel}}\left(t ; t_{\mathrm{ref}}\right)=\mathbf{x}_{\mathrm{obs}}(t)+\left(t_{\mathrm{ref}}-t\right) \mathbf{V}_{f},
$$

where $\mathbf{x}_{\mathrm{rel}}\left(t ; t_{\mathrm{ref}}\right)$ is the position of the shifted flight track corresponding to the observation made at $t$ and $\mathbf{V}_{f}$ is the velocity of the front. Finally, the aircraft observations are compared to model output at $t_{\text {ref }}$ interpolated to $\mathbf{x}_{\text {rel }}\left(t ; t_{\text {ref }}\right)$. Since the circuit BCDEF took just over an hour to complete, all quantities presented in the following have been evaluated twice, using model output at $t_{\text {ref }}=1500 \mathrm{UTC}$ and $t_{\text {ref }}=1600 \mathrm{UTC}$, and were found to be similar.

The front velocity $\mathbf{V}_{f}$ is estimated from the model simulations. If the front were homogeneous in the alongfront direction, then only the component of $\mathbf{V}_{f}$ perpendicular to the front would be required in (4). However, the front considered here exhibits substantial alongfront inhomogeneities (see below), so both alongfront and cross-front components of $\mathbf{V}_{f}$ are used. These are estimated by manually tracking vorticity anomalies on the front in the model simulations, and are found to be $14.2 \mathrm{~m} \mathrm{~s}^{-1}$ (alongfront speed) and $9.4 \mathrm{~m} \mathrm{~s}^{-1}$ (cross-front speed). These values are largely consistent between the model simulations, and the results presented here are not sensitive to the precise values used. Using the above-stated values for $\mathbf{V}_{f}$ with $t_{\text {ref }}=1500$ UTC gives the shifted flight track position indicated by the gray dotted line in Fig. 1.

\section{The observed cold front}

\section{a. Synoptic situation}

November 2009 was mild and exceptionally wet in the United Kingdom. An almost continuous chain of intense extratropical cyclones tracked across the North Atlantic toward the British Isles, resulting in strong winds and widespread heavy rainfall (Eden 2010). Most notably, the storms of 18-20 November resulted in what was at the time the wettest $24-\mathrm{h}$ period ever recorded at a location in the United Kingdom, in Borrowdale in northern England $(316 \mathrm{~mm})$, and the subsequent flooding of the town of Cockermouth, England (Eden and Burt 2010). The subject of the present paper is the cyclone that developed west of Ireland on 24 November and passed over Scotland on 25 November. A pressure minimum of $962 \mathrm{hPa}$ was attained at 0000 UTC $25 \mathrm{No}-$ vember, at which time its cold front was oriented southwest-northeast across the United Kingdom (see
Fig. 3a). While less intense than its predecessors earlier in the month, the impact of the precipitation was still felt, particularly in the northwest region of the United Kingdom, as a result of the ground being saturated from the earlier events.

The radar-derived precipitation rate associated with the cyclone and cold front at 1900 UTC 24 November 2009 is shown in Fig. 3b. During the evening of 24 November, the frontal precipitation advanced over Wales and England. It consisted of a broad band of moderate rainfall around $100 \mathrm{~km}$ wide with an NCFR of intense rainfall, on the order of $10 \mathrm{~km}$ wide, at its leading edge. The NCFR is not continuous along the front but rather split into discrete segments of precipitation separated by gaps where precipitation rates are low.

\section{b. In situ aircraft observations of frontal structure}

Measurements of horizontal wind, temperature, and humidity from the low-level legs $\mathrm{A}-\mathrm{G}$ are shown in Fig. 4. The track begins ahead of the front at point A from which it runs roughly parallel to the front at 40-m altitude before ascending to $300-\mathrm{m}$ altitude at point B and turning toward the front, which it crosses at 1510 UTC (transect BC). At point $\mathrm{C}$ it turns to run parallel with the front in the cold sector. At point $\mathrm{D}$ it turns back toward the front, which it crosses around 1545 UTC (transect DE). Finally, at point E the circuit turns to run parallel with the front in the warm sector. Point $\mathrm{F}$ is the point along the leg EG that is closest to point $\mathrm{B}$ in the front-relative frame of reference (see Fig. 1). Exact closure was not achieved because of the difficulty in forecasting the frontal velocity in real time; however, the error is small.

Away from the front, each of the four variables shown in Fig. 4 are roughly constant: there is a $30 \mathrm{~m} \mathrm{~s}^{-1}$ southsouthwesterly flow ahead of the front at $300 \mathrm{~m}$ and a $15 \mathrm{~m} \mathrm{~s}^{-1}$ westerly flow behind, and there is a temperature difference of $4 \mathrm{~K}$ between the air masses and a specific humidity difference of $1.5 \mathrm{~g} \mathrm{~kg}^{-1}$. Clark and Parker (2014) classify a series of NCFRs observed over the United Kingdom into three types, based on the magnitude of wind veer across the front and drop in wind speed. The strong reduction in wind speed across the front that is evident in Fig. 4 suggests this is their "type B" NCFR, which they suggest is unlikely to be tornadic.

In the vicinity of the front, there is a remarkable contrast between the frontal structure in the two transects. Transect BC exhibits a single sharp transition in wind velocity and temperature, with a change in the alongfront wind of $14 \mathrm{~m} \mathrm{~s}^{-1}$ over a horizontal distance of $600 \mathrm{~m}$, equating to a shear vorticity of $0.023 \mathrm{~s}^{-1}$. In transect DE both the wind direction and humidity fields 
(a)

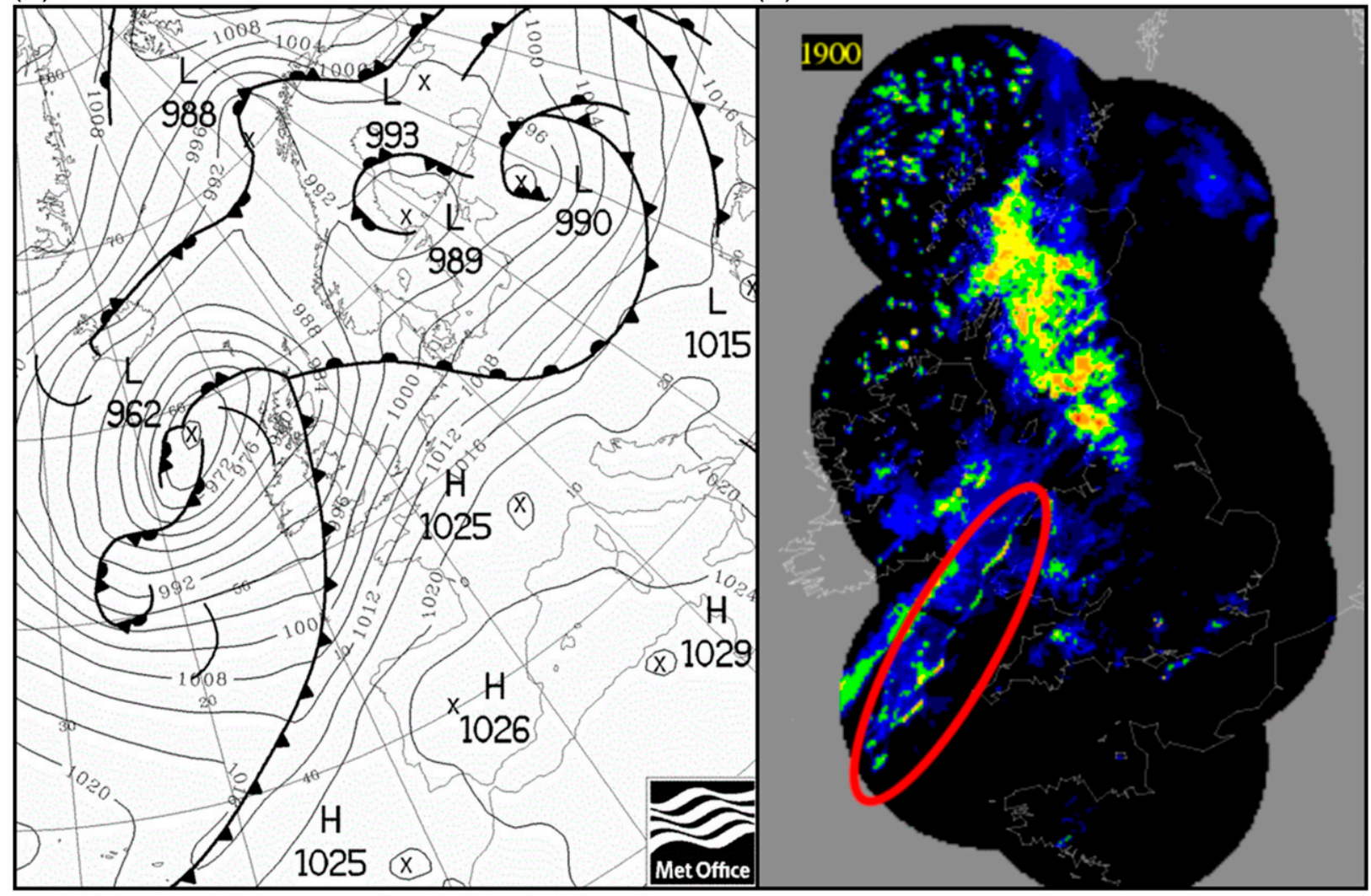

FIG. 3. (a) Met Office surface analysis at 0000 UTC 25 Nov 2009 and (b) Met Office radar-derived precipitation rates at 1900 UTC 24 Nov 2009. The positions of the NCFR are highlighted in the radar image.

exhibit two distinct transition regions spaced $12 \mathrm{~km}$ apart. Both transects also have spikes in the humidity field at the front crossings that are likely due to a combination of precipitation and the response time of the instruments.

\section{Quantitative evaluation of the frontal structure in the simulations}

An overview of the low-level horizontal structure of the front in the model simulations is presented in Fig. 5, which shows snapshots of the vertical component of relative vorticity and the horizontal divergence at 300-m altitude. These fields show clearly the presence of the front, the position and orientation of which are consistent between the simulations. Also evident in Fig. 5 is the NCFR instability in which the vorticity band along the front rolls up into isolated coherent vortices, connected by thin filaments of high vorticity and convergence (i.e., negative divergence).

While the front and the frontal instability are evident in each model simulation, there are systematic changes as the model resolution is increased. Most notably, it appears that as the model resolution increases, the width of the front decreases, the maximum magnitudes of the vorticity and divergence fields increase, the size of the coherent vortices decreases, and their alongfront
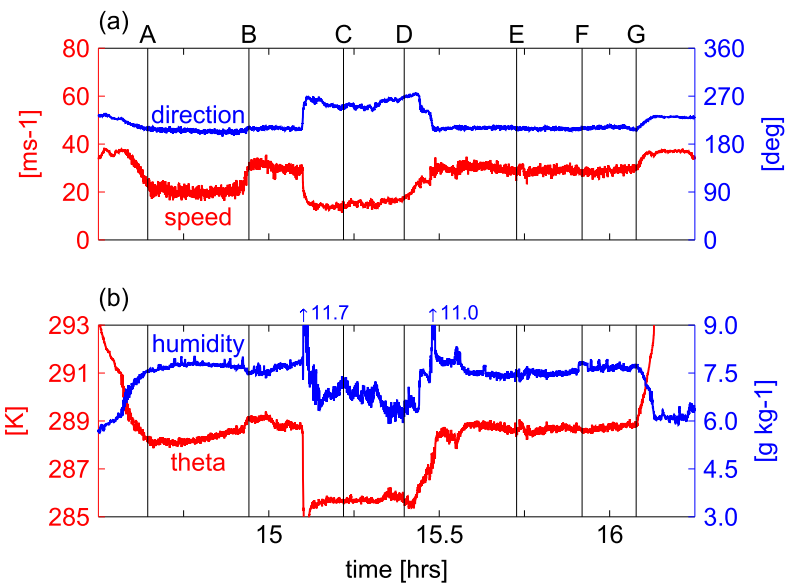

FIG. 4. Time series of observed (a) wind speed and direction, and (b) potential temperature and specific humidity. The aircraft altitude was $40 \mathrm{~m}$ during leg $\mathrm{AB}$ and $300 \mathrm{~m}$ during circuit $\mathrm{BG}$, with the ascent beginning 1 min before point $B$. The locations of points $A-G$ are indicated in Fig. 1. 

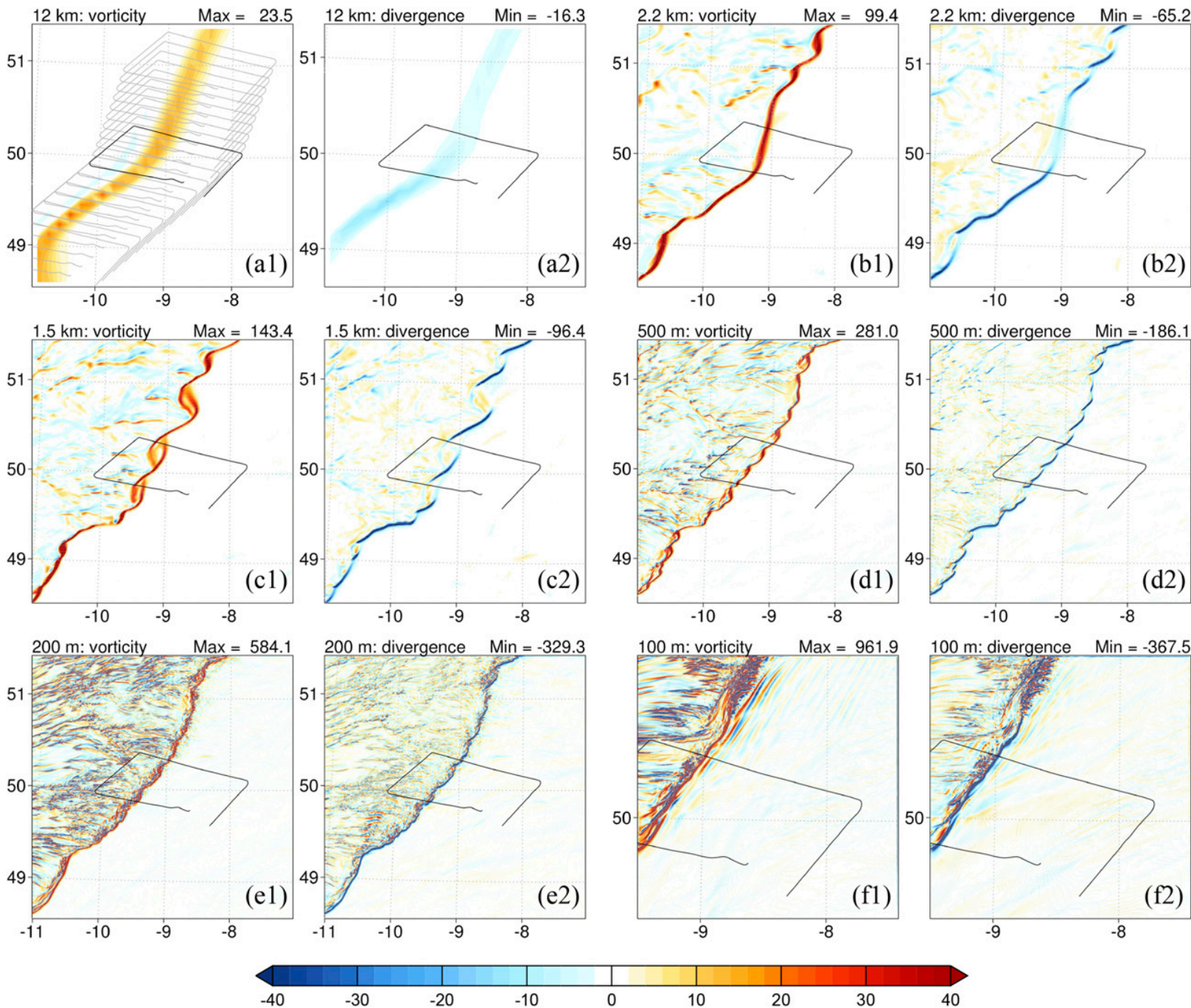

FIG. 5. Simulated relative vorticity ("1") and divergence ("2") in the vicinity of the front at 1500 UTC, altitude $300 \mathrm{~m}\left(10^{-4} \mathrm{~s}^{-1}\right)$. The simulations shown are (a) $12 \mathrm{~km}$, (b) $2.2 \mathrm{~km}$, (c) $1.5 \mathrm{~km}$, (d) $500 \mathrm{~m}$, (e) $200 \mathrm{~m}$, and (f) $100 \mathrm{~m}$. The 1500 UTC shifted flight track is indicated in all panels, and (a1) also shows the sample of 20 alternative circuits described in section $4 a$. Note that (f1) and (f2) show a smaller area, equal to the full domain used for the 100 -m simulations.

spacing decreases. In addition, there is substantial resolved convective activity behind the front, but not ahead of it, in the higher-resolution simulations. For a complementary overview of the frontal structure in the model simulations, Fig. 1 in the online supplemental material shows corresponding plots for potential temperature and specific humidity, and the same qualitative conclusions can be drawn from those variables.

In this section, the resolved frontal structures are compared quantitatively between the model simulations and the aircraft observations in order to assess the extent to which the model converges on the observed atmospheric characteristics with increasing resolution. First, the area-average vorticity and divergence across the front are calculated via circuit integrals. While the width of the front decreases with resolution, the magnitude of the maxima in the vorticity and divergence fields increases. The expectation is that the area-average values are insensitive to resolution, even if the finescale structure of the front is not well resolved. Second, the local structure of the front is analyzed. Do the highest-resolution model simulations capture the observed frontal width? Aspects relating to the turbulent activity seen in Fig. 5 are considered in section 5, and the characteristics of frontal instability are discussed in section 6 .

It is noted that Fig. 5 also shows evidence of influence from the domain boundary in the 200- and 100-m simulations, in which roll-like structures emanate from the proximate inflow (i.e., western) boundary (Figs. 5e and 5f). 
Such rolls are a common feature in $O(100)$ m models (Boutle et al. 2014). The rolls decay before reaching the area of the flight track in the $200-\mathrm{m}$ simulation, but not the $100-\mathrm{m}$ simulation, indicating that care is needed in drawing conclusions from that simulation. In addition, there are strong wave-type features immediately ahead of the front in the 100-m simulation, perhaps indicating that the frontal structure is adjusting to smaller scales in the 100-m simulation and emitting prefrontal gravity waves in the process (e.g., Shakespeare and Taylor 2014). A strong prefrontal gravity wave packet parallel to the front was observed from the aircraft, as described by Knippertz et al. (2010), but the similarity to the gravity waves evident in the $100-\mathrm{m}$ simulation is not explored further here. Finally, it is also noted that the 100-m simulation appears to exhibit a numerical instability lying along the front from where it leaves the domain at the northern boundary. Together, these shortcomings of the $100-\mathrm{m}$ simulation highlight the current limitations of trying to attain high-resolution simulations of nonstationary dynamic features such as fronts. Further, 100-m simulations with a larger domain will prove useful for understanding these processes, but they were not possible at the time of writing because of the computational cost.

\section{a. Integral measures of front intensity}

As discussed above, the design of the flight track as a closed circuit in a system-relative sense allows for direct evaluation of the area-average vorticity (VOR) and divergence (DIV) values within the circuit. The Stokes and Gauss theorems give

$$
\mathrm{VOR}=-\frac{1}{A} \oint_{\mathscr{C}} \mathbf{V} \cdot \mathbf{s} d l
$$

and

$$
\mathrm{DIV}=\frac{1}{A} \oint_{\mathscr{C}} \mathbf{V} \cdot \mathbf{n} d l
$$

respectively, where $\mathbf{V}$ is the horizontal wind; $\mathscr{C}$ is the shifted front-relative flight track; $\mathbf{s}$ and $\mathbf{n}$ are unit vectors pointing along and perpendicular to the left of the flight track, respectively; $A$ is the area enclosed by $\mathscr{C}$; and $l$ is the distance around the perimeter of $\mathscr{C}$ measured in the direction of the flight. Note that the shifted flight track is the appropriate contour for both the model data and the observations, despite the observations being taken along the unshifted flight track, because it is equivalent to viewing observations in a front-relative frame under the assumption that the finescale structure of the front is frozen in time. The negative sign in (5) arises because the aircraft flew clockwise around the circuit, whereas the mathematical convention is that circuit integrals are performed counterclockwise.

Simpler bulk estimates for shear vorticity and divergence are commonly calculated from differences in the mean alongfront and cross-front wind components ahead and behind the front. This calculation is also performed here to explore the impact of using the exact circuit integral expressions. Assuming that the flight track is parallel to the front along the segments $\mathrm{CD}$ and $\mathrm{EF}$, which was the aim of the flight plan but cannot be verified exactly, these bulk estimates of vorticity and divergence take the form

$$
\mathrm{VOR}_{\text {bulk }}=-\frac{1}{d}\left(\overline{\mathbf{V} \cdot \mathbf{s}}^{\mathrm{CD}}+\overline{\mathbf{V} \cdot \mathbf{s}}^{\mathrm{EF}}\right)
$$

and

$$
\mathrm{DIV}_{\text {bulk }}=-\frac{1}{d}\left(\overline{\mathbf{V} \cdot \mathbf{n}}^{\mathrm{CD}}+\overline{\mathbf{V} \cdot \mathbf{n}}^{\mathrm{EF}}\right)
$$

respectively, where $d$ is the distance between the middle of the alongfront segments $\mathrm{CD}$ and $\mathrm{EF}$, and the overbars indicate averages along the flight segments indicated. The similarity with the exact contour integral expressions (5) and (6) is apparent. The key differences are that the bulk estimates (i) assume the flight track is parallel to the front and (ii) neglect the contributions from the cross-front transects BC and DE.

Figure 6 shows the contour integrals [(5) and (6)] (Fig. 6a) and the bulk estimates [(7) and (8)] (Fig. 6b), all of which are calculated from the observations and all model simulations except for the $100-\mathrm{m}$ resolution, since the flight track exceeds the limits of the simulation domain in that case. In the observations, both vorticity diagnostics are positive and both divergence diagnostics are negative, indicative of cyclonic shear and convergence at the front, respectively. The magnitudes of all four values are very similar at around $1 \times 10^{-4} \mathrm{~s}^{-1}$. The fact the contour integrals and bulk estimates are similar suggests that the flight track is indeed parallel to the front. Note that these are smaller in magnitude than typical point values of vorticity and divergence at fronts because they represent an area average over the flight circuit region, which is considerably broader than the front itself (see Fig. 5).

The values have been calculated from both 1500 and 1600 UTC model outputs and were found to be largely similar. However, Fig. 5 shows that there are substantial alongfront inhomogeneities in the vorticity and divergence fields associated with the frontal instability in the model, and these can be expected to show up in the area-average diagnostics. If these features are realistic, 

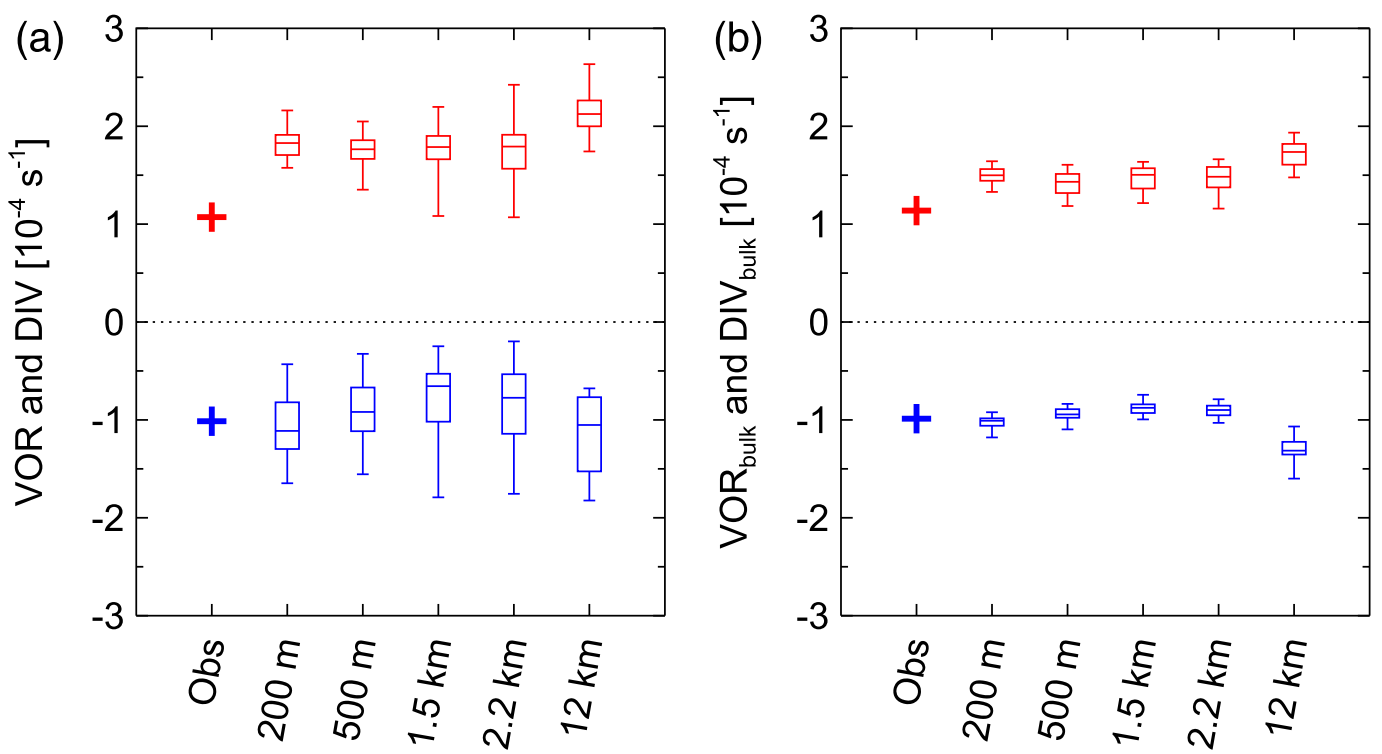

FIG. 6. Area-average vorticity (red) and divergence (blue) values from (a) the exact expressions of (5) and (6), and (b) the bulk estimates of (7) and (8). The plus symbols indicate the observed values, and the box-and-whisker symbols indicate the median, interquartile range, and full range of the sample of 42 model circuits consisting of the original flight track and the 20 alternative circuits, all evaluated from both 1500 and 1600 UTC data model outputs. The 100-m simulation is not included, as the domain used does not cover the entire flight track region, and the 200-m simulation has a sample of only 30 circuits as a result of some lying outside of the domain.

then the observed values will vary depending on the precise location of the flight track. While the scale and structure of the frontal instability may be captured by the model simulations, the locations of the frontal segments are likely to have little predictability. Therefore, the alongfront inhomogeneity amounts to a sampling uncertainty in the observations. To estimate this uncertainty, the vorticity and divergence diagnostics [(5)-(8)] are recalculated for a sample of 20 alternative flight tracks, identical to the actual flight track except for a translation parallel to the front of up to $120 \mathrm{~km}$, and the distribution of values obtained are indicated by the box-and-whisker symbols in Fig. 6. In total there are 42 values calculated (from the original circuit plus 20 alternative circuits at both 1500 and 1600 UTC), except for the $200-\mathrm{m}$ simulation, where 12 of the alternative circuits exceed the limits of the simulation domain so are excluded, leaving a sample of 30 values. The locations of the 1500 UTC sample of shifted flight tracks are shown in Fig. 5a for reference.

Both divergence diagnostics are consistent with the observations, in the sense that the observed values lie within the sample spread of the simulations, at all resolutions except $12 \mathrm{~km}$. In that simulation the bulk estimate is larger in magnitude than the observation. The sampling uncertainty is substantially larger for the contour integrals than the bulk estimates, indicating that the cross-front transects $\mathrm{BC}$ and $\mathrm{DE}$ are contributing to the variability of the area-average diagnostics. In contrast, the vorticity diagnostics are less consistent between the simulations and the observations: the mean vorticity values from all model circuits is larger than the observations in all simulations, with only the lowest vorticity values from the $1.5-$ and $2.2-\mathrm{km}$ simulations encompassing the observed value. Interestingly, the mean of the sample does not vary much with resolution but the sample spread does, with the 1.5- and 2.2-km simulations exhibiting the largest alongfront inhomogeneity. This is consistent with the larger vortices exhibited by those two simulations in Fig. 5.

The mismatch between the observed and simulated vorticity diagnostics in all but the 1.5 - and $2.2-\mathrm{km}$ simulations could be due to errors in the initial and boundary conditions from the global model acting to produce a front that is too strong in all simulations. Alternatively, it may be that the alongfront inhomogeneity is underrepresented in the highestresolution simulations. It is clear from Fig. 5 that the 2.2- and $1.5-\mathrm{km}$ simulations exhibit larger vortices than the other simulations, and this is consistent with the large sample spread evident in those simulations in Fig. 6a. We hypothesize that the low vorticity value in the observations relative to the model simulations may be the result of the chance positioning of the flight circuit relative to the alongfront rainbands, leading to a value from near the low end of the sample spread, combined 
with the fact that the vortices are too small in the subkilometer simulations. This hypothesis is explored further below.

\section{b. Cross-front transects}

Although a full picture of the actual alongfront structure cannot be inferred from the two observed transects alone, the difference between the frontal structure in the two observed transects (see Fig. 4) provides strong evidence for the influence of NCFRs. It appears that transect $\mathrm{BC}$ passes through a narrow filament of strong vorticity and convergence of width $600 \mathrm{~m}$, whereas transect DE crosses the core of a vortex with a diameter of at least $12 \mathrm{~km}$, with the two transition regions located at the edges of the vortex. This interpretation is now examined further by comparison with the model simulations.

To compare the observed cross-front transects with the model simulations, the alongfront and cross-front wind components are calculated as

$$
U_{\text {para }}=\mathbf{V} \cdot \overline{\mathbf{n}}^{\mathrm{CD}}
$$

and

$$
U_{\text {perp }}=\mathbf{V} \cdot \overline{\mathbf{s}}^{\mathrm{CD}},
$$

respectively, where $\overline{\mathbf{s}}^{\mathrm{CD}}$ and $\overline{\mathbf{n}}^{\mathrm{CD}}$ are the unit vectors along and perpendicular to the left of the flight track averaged along transect $\mathrm{CD}$. Figure 7 shows profiles of $U_{\text {para }}$ from the two observed transects (Fig. 7a) and the 42 sample circuits for each model simulation (Figs. 7c-h). The cross-front wind $U_{\text {perp }}$ from the two observed transects is also shown (Fig. 7b). All of the profiles have been shifted so that they align where $U_{\text {para }}=25 \mathrm{~m} \mathrm{~s}^{-1}$, for clarity, since the position of the front relative to the circuits is not identical in each simulation.

The difference between the two observed transects in Fig. 7a is striking, indicating that the double-step structure in the wind direction along transect DE is associated with a similar structure in the alongfront wind speed. The two cross-front wind speed transects are more similar to each other, with most of the convergence at the leading edge of the front in both cases. In contrast to the observations, the $12-\mathrm{km}$ simulation exhibits a relatively smooth transition across the front of alongfront wind speed over a distance of around $20 \mathrm{~km}$, which is larger than observed in either transect, with little variation along the front. As the resolution is increased, the gradient of the alongfront wind speed at the front increases, with the three highest-resolution simulations capturing the width of the sharp transition observed in transect $\mathrm{BC}$ (see below). Out of all the model simulations, the presence of a double-step structure of a separation of $12 \mathrm{~km}$ is captured only by the $1.5-\mathrm{km}$ simulation and partially by the $2.2-\mathrm{km}$ simulation, consistent with these simulations having the largest vortices present in Fig. 5.

To test the hypothesis that the chance positioning of the observed circuit has led to a relatively low value of the vorticity integral, the sample circuit with the lowest vorticity value from the $1.5-\mathrm{km}$ simulation is highlighted in Fig. 7e. There is a strong similarity with the aircraft observation, with the first transect consisting of a single sharp jump in wind speed and the second transect exhibiting a double-step structure. While it is expected that the relationship between the transect wind speeds and the area-average vorticity values is nontrivial, the fact that the positioning of the sample circuit with the lowest vorticity values appears similar to the observed circuit provides evidence that the observed circuit may encompass lower vorticity than elsewhere along the front.

For completeness, Fig. 2 in the online supplemental material shows the corresponding transects of potential temperature from the observations and the model simulations. Interestingly, for transect DE the potential temperature structure is very different from the alongfront wind, but it has similarities with the cross-front wind. As the model resolution increases, the maximum potential temperature gradient at the front increases, and again the most realistic vortex structures are found in the $1.5-\mathrm{km}$ simulation. Furthermore, the highlighted circuit in Fig. 2e in the supplemental material, which again corresponds to the model circuit with the smallest vorticity value, shows some similarity to the observed transects. The main difference is a temperature maximum at around $-15 \mathrm{~km}$ in the second transect that is not present in the observations. Figure 1 in the supplemental material shows that there is substantial structure in the potential temperature field associated with the vortices in the $1.5-\mathrm{km}$ simulation, including temperature maxima in the centers of some of the vortices. The fact that the observed transect does not exhibit such a maxima is likely due to the precise positioning of the aircraft circuit relative to the vortex center.

Also apparent in Fig. 7 is the presence of overshoots in the alongfront wind speed at the model-simulated fronts. These are particularly clear in the 1.5-, 2.2-, and $12-\mathrm{km}$ simulations (where $U_{\text {para }}<0$, for instance), although they can be seen to some extent in all simulations. The overshoots are not present in the observed transects, suggesting that they are a numerical artifact. Such overshoots are common near regions of strong gradients in (nonmonotonic) semi-Lagrangian advection schemes, and they may indicate that the advection scheme 

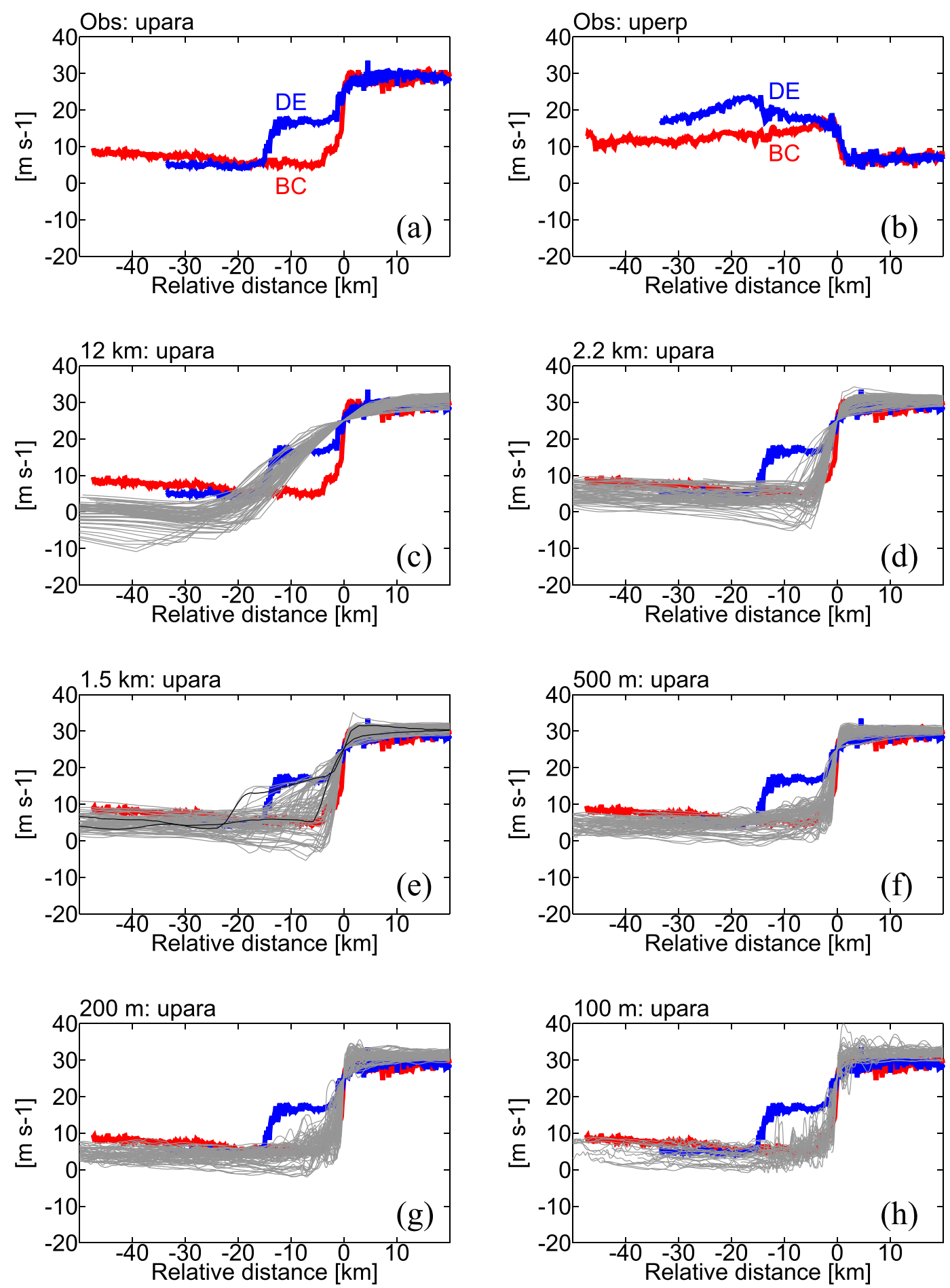

FIG. 7. (a) Observed alongfront wind profiles from flight legs BC (red) and DE (blue). (b) Observed cross-front wind profiles from flight legs BC (red) and DE (blue). (c)-(h) Model profiles of alongfront wind from the simulations and showing one of the samples of the model circuits (see text; gray), and the red and blue lines are as in (a). In addition, in (e) the sample circuit exhibiting the lowest area-average vorticity value is indicated (black). In all panels the profiles are shifted spatially to align the front as described in the text. 

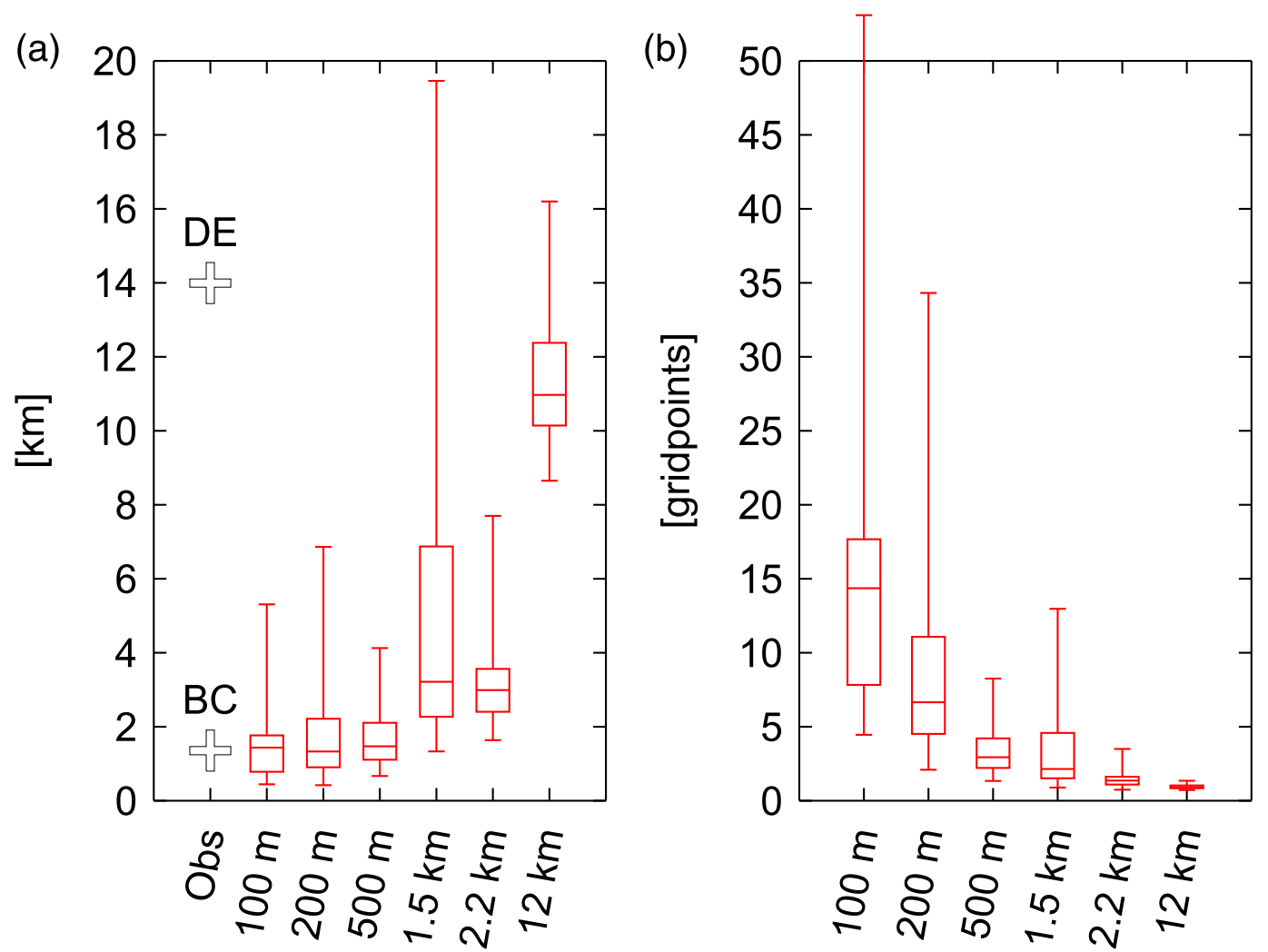

FIG. 8. Frontal widths, calculated as described in the text, in (a) kilometers and (b) grid points. The box-andwhisker symbols indicate the median, interquartile range, and full range from the sample of circuits. The crosses in (a) are the observed values from the two transects, as indicated.

numerics are playing a role in limiting the frontal collapse rather than other more physical processes. This hypothesis is discussed further in section 7. The overshoots are less evident in the 500-, 200-, and 100-m simulations, perhaps indicating that the frontal width is not being limited by the advection scheme numerics in those cases.

To infer further information about the variation of the frontal gradients with resolution, a simple measure of frontal width is defined as the minimum distance between points with $U_{\text {para }}=12 \mathrm{~m} \mathrm{~s}^{-1}$ and $U_{\text {para }}=25 \mathrm{~m} \mathrm{~s}^{-1}$ in Fig. 7. This definition of frontal width is inversely proportional to the bulk wind shear at the front. The threshold values were chosen subjectively in order to capture the clear double-step feature in the observations. However, the results are not qualitatively sensitive to the precise values used, nor are they sensitive to using thresholds based on the temperature transects instead of wind speed. The frontal width values are shown in units of physical distance in Fig. 8a and scaled by the model grid spacing in Fig. 8 b. As previously, the two contrasting observed values are indicated by the crosses, and the box-and-whisker symbols illustrate the distribution from the sample of circuits from each simulation. Following the discussion above, the lower end of the sample bars is interpreted as the scale of the narrowest PV filaments in each simulation, whereas the upper end represents the width of the widest vortex. The three highest-resolution simulations encompass the narrow observation (transect BC), but do not exhibit frontal widths as large as the wide observation (transect DE). Therefore, while the high-resolution simulations are able to capture the remarkably sharp gradients observed at the front, and indeed these appear to be well resolved in the 200- and 100-m simulations (Fig. 8b), they do not produce vortices with core widths as large as observed by the research aircraft. Only the $1.5-\mathrm{km}$ simulation comes close to encompassing both the high and low observed width values, and as such it appears to have the most realistic representation of both the frontal width and the frontal shear instability.

\section{Turbulent fluxes}

Figure 5 shows that resolved turbulent activity becomes more active in the cold sector at high resolution. In contrast, there is much less resolved turbulent activity 
(a) sensible heat flux



(c) wind stress

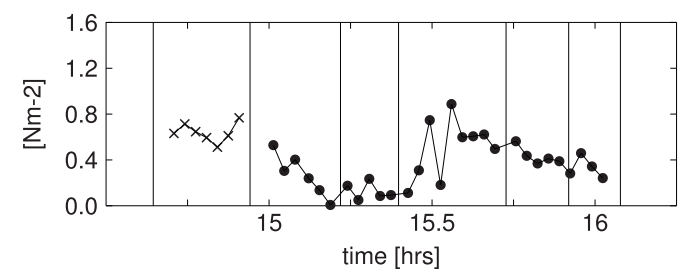

(b) latent heat flux



(d) TKE



FIG. 9. Time series of (a) sensible heat flux, (b) latent heat flux, (c) wind stress, and (d) TKE. Each dot (cross) represents the eddy covariances calculated from $32-\mathrm{Hz}$ data over straight and level 2-min legs at 300-m (40 m) altitude, as described in the text.

in the warm sector, even in the 100-m simulation, despite the observed wind speed in Fig. 4 being most variable there. This highlights the difference in boundary layer regimes in the two regions: there is intense shear-driven turbulence present in the warm sector ahead of the front but at scales too small to be resolved in the simulations, whereas the boundary layer in the cold sector is convectively unstable and there are overturning circulations present of a similar scale to the boundary layer depth. This case therefore provides a challenging test for the turbulence parameterization in the model. In this section both the resolved and subgrid-scale parameterized turbulent fluxes from the model simulations are evaluated against the observations.

Values of the sensible and latent heat fluxes, wind stress, and turbulent kinetic energy (TKE) have been calculated from the aircraft observations, as described in section 2a. Time series of the observation-derived fluxes are shown in Fig. 9, in which each dot represents a single straight and level 2-min leg. In the warm sector (legs AB and $E G)$, there is a downward sensible heat flux, an upward wind stress, and an upward latent heat flux. These are as expected because of the advection of relatively warm air over a cooler ocean. In the cold sector (leg CD), both the sensible heat flux and the wind stress are much smaller, whereas the latent heat flux is similar in magnitude to its prefrontal value. Typically, surface sensible and latent heat fluxes are large and positive in cold-sector air. This is not the case here, presumably because the fluxes are measured at 300-m altitude rather than the surface. Apart from the fluxes in the vicinity of the front itself, the values of all the fluxes are roughly constant. As such, for comparison with the model simulations, attention is now restricted to the average values along the legs $\mathrm{AB}, \mathrm{CD}$, and $\mathrm{EG}$.

Turbulent fluxes in the model simulations are computed as the sum of the resolved and parameterized components. The resolved component is computed following the same method as the observations, by first interpolating variables onto the shifted flight track, and then using the same straight and level 2-min runs as the observations to compute covariance values. The parameterized component is taken directly from the relevant parameterization schemes and is likewise interpolated onto each 2-min run. Figure 10 shows these values calculated for each model simulation. Indicated in the figure are the observations (crosses), and the resolved (blue), parameterized (red), and total (black) fluxes for each model simulation. The box-and-whisker symbols indicate the spread of leg-average model values from the sample of circuits, whereas the small crosses indicate the range of individual 2-min run values in the observations.

Considering first the cold-sector leg $\mathrm{CD}$, both the latent heat flux and the wind stress exhibit a gradual transition from being fully parameterized in the $12-\mathrm{km}$ simulation to around $80 \%$ resolved at $100-\mathrm{m}$ resolution. To within the sample spread, the sum of the resolved and parameterized components remains constant with varying resolution and is consistent with the observed values. The subgrid turbulence scheme is therefore successfully accounting for the partially resolved eddies in this case. Likewise, the TKE converges, although the sampling variability becomes very large in the $100-\mathrm{m}$ simulation. The sensible heat flux, in contrast, does not hand over monotonically from parameterized to 

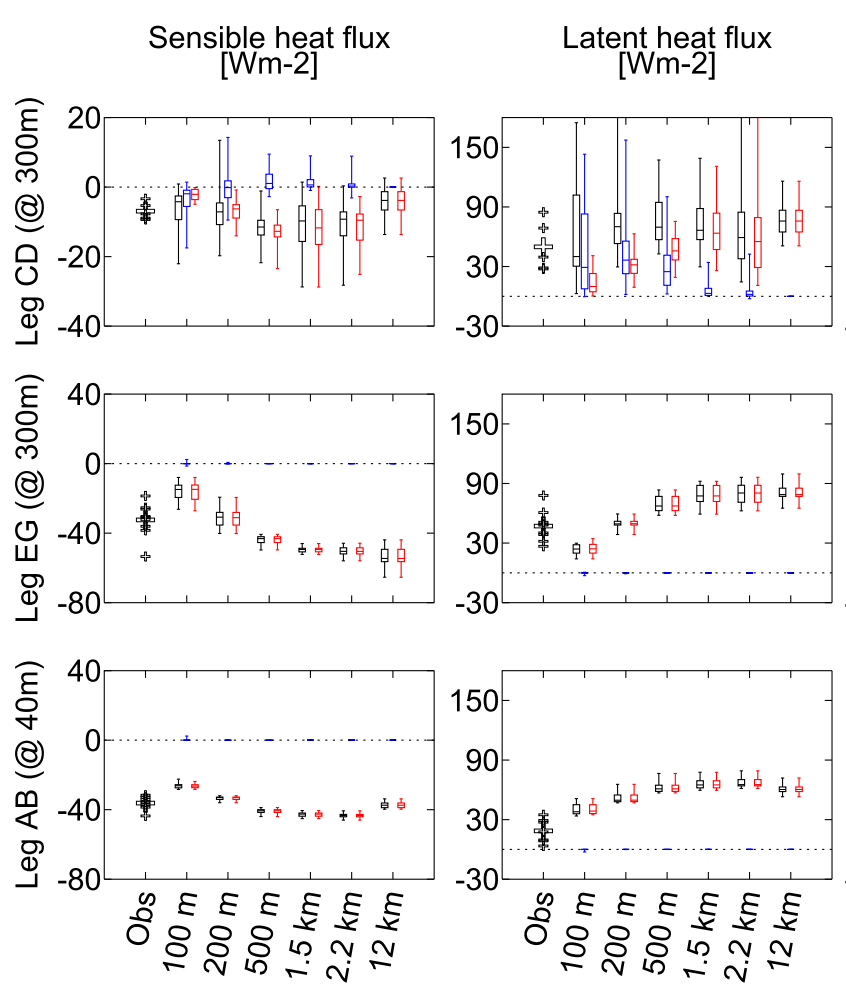

Wind stress

[Nm-2]
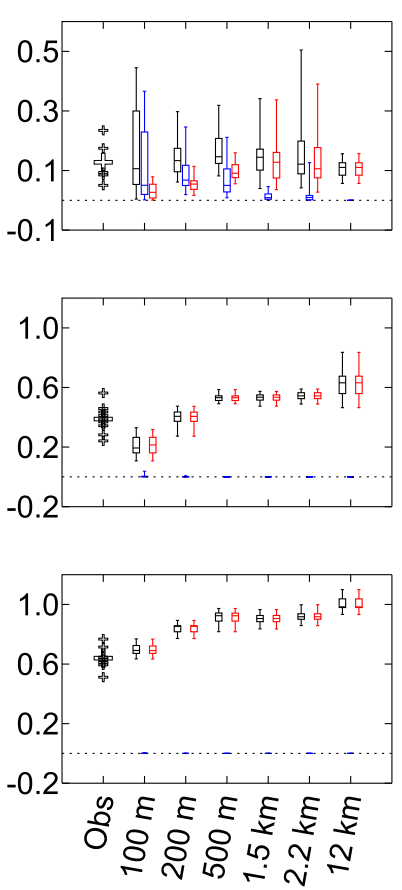

TKE

$[\mathrm{m} 2 \mathrm{~s}-2]$
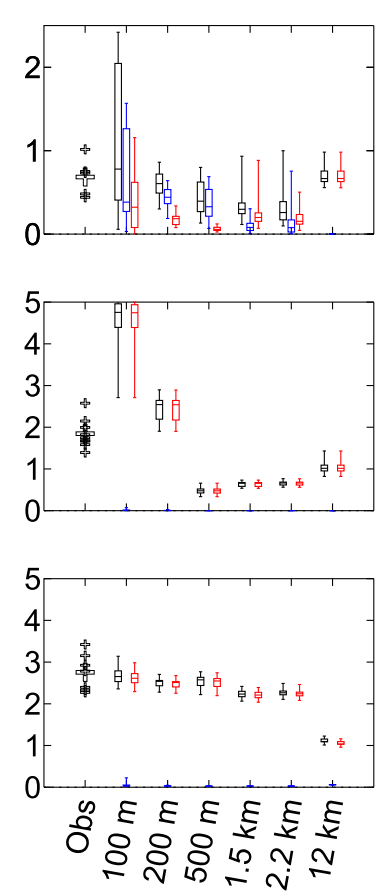

FIG. 10. Summary of observed (crosses) and simulated (box and whisker) turbulent fluxes along (top) the cold side of front at $300 \mathrm{~m}$, (middle) the warm side of front at $300 \mathrm{~m}$, and (bottom) the warm side of front at $40 \mathrm{~m}$. For the observations the large cross shows the legaverage value and the small crosses show the values of the individual 2-min runs. For the model simulations, the three bars indicate the median, interquartile range, and full range of leg-average values from the sample of circuits for the parameterized (red), resolved (blue), and total (parameterized plus resolved; black) fluxes.

resolved with increasing resolution. The resolved component is essentially zero in all simulations except $100 \mathrm{~m}$, whereas the parameterized component has the largest magnitude in the $1.5-\mathrm{km}$ simulations.

In the warm sector, the resolved fluxes are small in all model simulations at both 40-m altitude (leg AB) and 300-m altitude (leg EG), as anticipated from the lack of resolved turbulent activity in Fig. 5. The simulations that apply the nonlocal 1D boundary layer scheme (those with grid spacing $\geq 1.5 \mathrm{~km}$ ) show remarkably similar values for all three fluxes, and at both altitudes. However, in each case the magnitudes of all three fluxes are larger than observed. The TKE, in contrast, is smaller than the observed values and jumps between the 2.2and $12-\mathrm{km}$ simulations. Switching from the 1D boundary layer scheme to the 3D Smagorinsky scheme at 500-m resolution does not affect the fluxes much, but as the resolution is increased further the magnitudes of all fluxes decrease substantially. At 40-m altitude (leg AB) the effect is to move the model fluxes closer to the observed values, and the $100-\mathrm{m}$ simulation agrees closely with the observed values, except perhaps the sensible heat flux. However, the values do not appear to have converged by $100 \mathrm{~m}$, and the large jump between the $100-$ and $200-\mathrm{m}$ values suggests they may continue to decrease, beyond the observed values, if the resolution is increased further. At $300-\mathrm{m}$ altitude, this is precisely what happens: the fluxes reduce in magnitude as the resolution is increased and end up smaller than the observed values in the $100-\mathrm{m}$ simulation. In that case, the parameterized fluxes are closest to the observations in the 200-m simulation. It is of note that Stein et al. (2015) likewise found the 200-m configuration performed best when comparing the width of convective updrafts in this model with radar observations; however, there is no clear reason for assuming that the results are related in the different dynamical regimes.

A possible explanation for the poor performance of the 3D Smagorinsky scheme ahead of the front is that the scheme assumes the presence of a partially resolved inertial cascade. This is not the case here, since the shear-driven turbulence is subgrid, even at $100-\mathrm{m}$ grid spacing (see Fig. 5). In effect, the scheme is appropriate in the surface layer, which encompasses the 40-m observations, but not at higher altitude in the boundary layer. Physically, the mixing length is chosen to be 
proportional to the grid length in these simulations. But since grid-scale eddies are not present ahead of the front, the magnitude of the resolved shear does not increase with resolution. Therefore, the parameterized fluxes can be expected to decrease in line with the mixing length. The study of Boutle et al. (2014) introduced a pragmatic blending methodology in which a linear combination of the local and nonlocal mixing schemes is objectively selected based on environmental conditions, and it is expected that such a scheme may act to alleviate the unrealistic fluxes found ahead of the front here.

\section{Frontal instability and structure of the NCFRs}

While the aircraft observations provide high-resolution information on the cross-frontal structure and turbulent fluxes, the alongfront length scale of the NCFRs cannot be inferred from the aircraft observations alone. However, the NCFRs are clearly visible in the radar image of Fig. 3, albeit at a later time than the aircraft observations, and their alongfront length scale can be measured manually as the average distance between breaks in the NCFRs. This process has been repeated for each hour from the time the front enters the radar domain (1800 UTC). At each hour all of the NCFRs visible in the radar domain (around six) are used to compute the average wavelength. A similar process has been performed on the precipitation fields from the model simulations and the resulting wavelengths are summarized in Fig. 11.

The radar images exhibit an average wavelength of $100 \mathrm{~km}$, which remains roughly constant over time. In contrast, the $12-\mathrm{km}$ simulation has wavelengths that are too long (around $300 \mathrm{~km}$ ) and the subkilometer simulations exhibit wavelengths that are too short (around 40 and $20 \mathrm{~km}$, respectively). The $2.2-$ and $1.5-\mathrm{km}$ simulations, however, have similar wavelengths to those seen in the radar image (around $100 \mathrm{~km}$ ). Taken together with the results of section $4 \mathrm{~b}$, in which it was shown that the width of the vortices in the subkilometer simulations are too small, Fig. 11 provides evidence that the structure of the instability in the subkilometer models is indeed collapsing to a scale that is smaller than observed.

It is of note that Fig. 11 shows the wavelength in the $500-\mathrm{m}$ simulation increasing during the final $3 \mathrm{~h}$ of the simulation, toward a value closer to that observed. The corresponding precipitation maps are shown in Fig. 12 in which the small scale of the NCFRs at 1500 UTC in the 500-m simulation is evident (comparing Figs. 12a and 12b), together with the increase in scale at 2300 UTC (Fig. 12f). The reason for this increase in scale

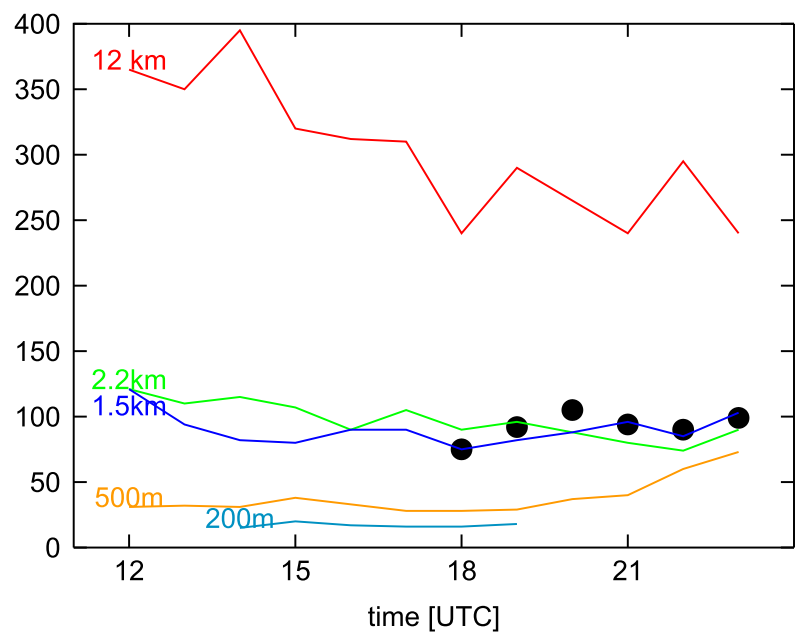

FIG. 11. The evolution of NCFR spacing in the radar images (black dots) and the five simulations (lines). The rainbands are outside of the radar range before 1800 UTC.

is not clear. One hypothesis is that a change in environmental conditions in the vicinity of the front over time leads to a change in the properties of the instability later in the simulation. Alternatively, the structure of the front may still be equilibrating to the higher resolution of the nested 500-m domain at 2300 UTC. It takes around $12 \mathrm{~h}$ for the vortical structures to spin up at the start of the 2.2-km simulation (not shown), which is similar to the time taken for the front to cross the 500-m domain.

Investigating further the dynamics of the NCFR instability in the subkilometer simulations is beyond the scope of this work. However, it is noted that other studies have been able to produce more realistic simulations of similar events using models of comparable resolution (e.g., Smart and Browning 2009; Apsley et al. 2016), albeit on smaller domains and therefore with less time for the high-resolution dynamics to modify the initial state taken from the coarser-resolution parent model. It is also noted that moist-frontal instability was studied in an idealized setting by Kawashima (2011), who suggests the nature of the instability is sensitive to the environmental conditions ahead of the front. They show that the NCFR instability can be stabilized by reducing the ambient cross-frontal shear, and that if there is sufficient environmental CAPE, then instead of NCFRs, the fastest-growing instability is convective in nature with a much smaller alongfront scale. This provides yet another hypothesis for the shift in the instability to small scales in the high-resolution simulations, in which systematic biases in the turbulent fluxes ahead of the front impact the frontal instability via this mechanism. These aspects of the dynamics will be investigated in a future study. 

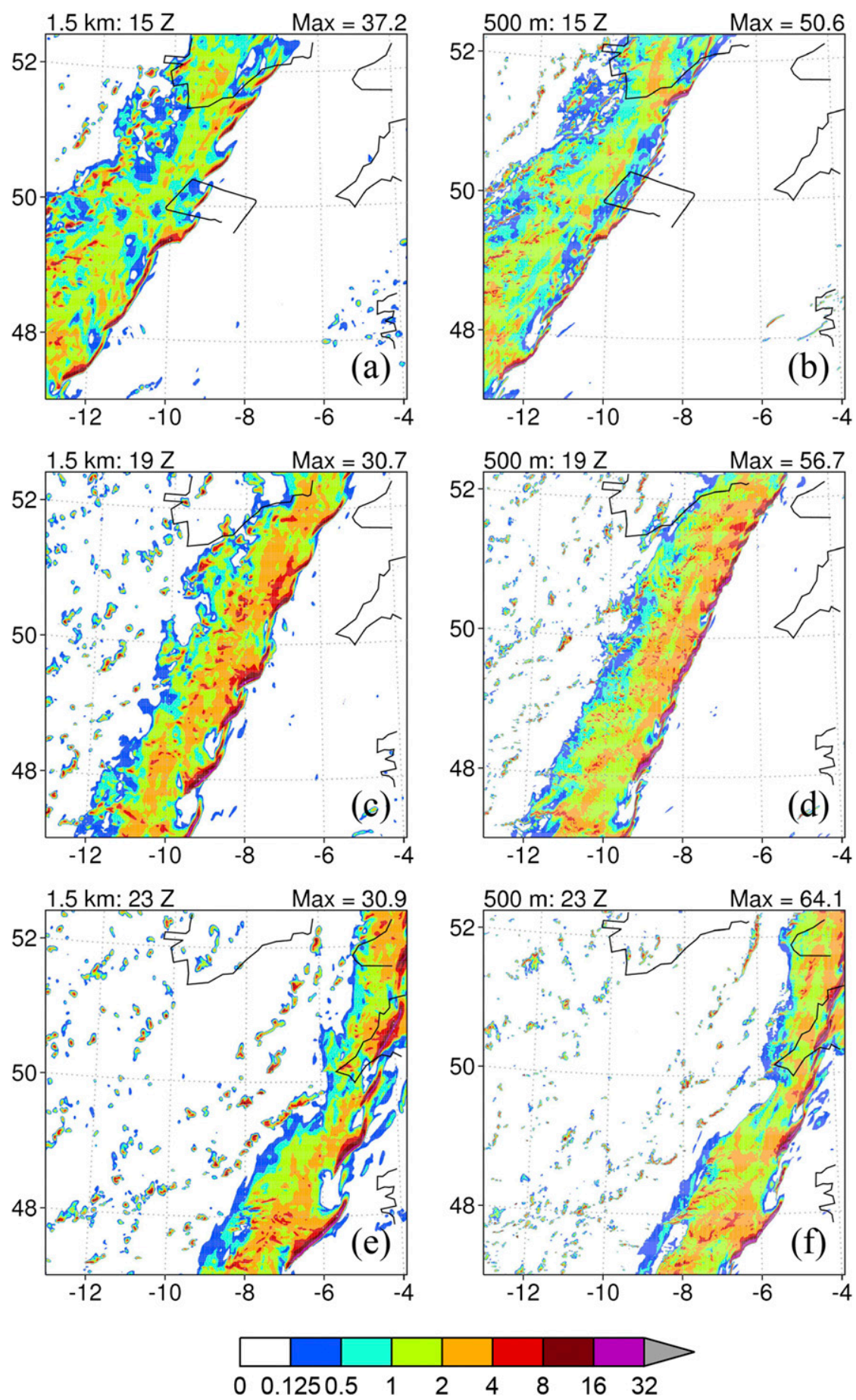

FIG. 12. Precipitation rate $\left(\mathrm{mm} \mathrm{h}^{-1}\right)$ in the (a),(c),(e) $1.5-\mathrm{km}$ simulation and (b),(d),(f) 500-m simulation at 1500, 1900, and 2300 UTC, respectively. The 1500 UTC shifted flight track is indicated in (a) and (b).

\section{Conclusions and discussion}

In situ aircraft observations are used to interrogate the convergence with the resolution of simulations of a narrow cold front. Simulations are performed with the
Met Office UM at a range of nested resolutions from grid spacings of $12 \mathrm{~km}$ to $100 \mathrm{~m}$. The observational strategy employed enables a comparison of flow structure over a broad range of scales, from the scale of the aircraft circuit $(80 \mathrm{~km} \times 140 \mathrm{~km})$ to small-scale turbulent 
motions as measured by $32-\mathrm{Hz}$ instruments (approximately $3 \mathrm{~m}$ ). Integral measures of the wind field are employed together with velocity gradients and turbulent fluxes to provide a comprehensive picture of the front across a wide range of scales.

The low-altitude horizontal divergence at the front successfully converges by $2.2-\mathrm{km}$ resolution, consistent with the frontal convergence field being driven by the cross-frontal circulation and relatively insensitive to the small-scale details at the front itself. The simulated lowaltitude vorticity is consistent only with the observations if the sampling uncertainty associated with the position of $100-\mathrm{km}$ precipitation segments along the front is accounted for. The model also converges on the observed frontal width where it is narrowest $(600 \mathrm{~m})$ in the highest-resolution simulations. These results imply that the net ascent out of the boundary layer at the front is also well represented.

The presence of overshoots in the alongfront wind behind the front is evidence that at lower resolution (1.5 km and coarser), implicit diffusion from the semiLagrangian advection scheme is limiting the frontal width. Repeated use of interpolation at back-trajectory departure points introduces diffusive-like effects, with the leading-order influence resembling $\nabla^{2}$ for linear interpolation and $\nabla^{4}$ for cubic interpolation (e.g., Harvey 2011). Whereas $\nabla^{2}$ diffusion acts to smooth a region of large gradient without exceeding the bounds of the initial field, hyperdiffusion of the form $\nabla^{2 n}$ with $n>1$ acts to smooth the regions of large gradient but can exceed the bounds of the initial field. In particular, when applied to a step function (an approximation to the alongfront wind field), such hyperdiffusion inevitably leads to overshoots (Mariotti et al. 1994). The horizontal scale of the overshoots should scale with the grid spacing, but the magnitude of the overshoots is independent of resolution, being a function only of the form of interpolation used. Their presence here suggests that the advection scheme numerics are playing a role in limiting the frontal collapse. The overshoots are less evident in the 500-, 200-, and 100-m simulations, perhaps indicating that the frontal width is not being limited by the advection scheme numerics in those cases.

In the convective boundary layer on the cold side of the front, the sum of the parameterized and resolved vertical eddy fluxes is approximately constant as the resolution increases and the parameterized fluxes hand over to resolved motions. The values are consistent with the aircraft observations, indicating a good representation of the turbulent gray zone in the convective boundary layer regime. However, on the warm side of the front where the boundary layer is stably stratified and the turbulence is shear driven, the model fluxes are entirely subgrid scale and the values are consistent only with observations from a 40-m-altitude flight leg. Poor performance is found from a $300-\mathrm{m}$ altitude flight leg in the sub-1-km-resolution simulations, perhaps as a result of a switch from a 1D boundary layer scheme to a 3D turbulent-mixing scheme more suited to Monin-Obukhovtype boundary layers.

Despite success in the simulation of frontal width and turbulent fluxes, the simulation of the vortex rollup along the front and the development of narrow coldfrontal rainbands do not converge. Large vortices consistent with the observations appear only in the $1.5-\mathrm{km}$ simulation, and to some extent in the $2.2-\mathrm{km}$ simulation, but not in the sub-1-km simulations. The reason for this is not clear; however, one hypothesis is that the transition between two types of instability is sensitive to initial conditions and the model parameterizations, giving alongfront instability low predictability. This result is unfortunate since such frontal rollup can be associated with high-impact weather events. For example, tornadoes typically occur in the British Isles in the "braids" joining the vortices (Clark and Parker 2014; Mulder and Schultz 2015). The prediction of the frontal instability is therefore a challenge for forecasting high-impact weather, and the results presented here emphasize that model resolution alone is not sufficient for success. Future work will aim to understand the nature of the instability in more detail and the reasons for the collapse to smaller scales in the subkilometer simulations.

The results shown here also demonstrate the utility of the observational strategy employed. The use of a closed-circuit flight track in the frame of reference of the front enabled the accurate calculation of area-average vorticity and divergence values, although the substantial alongfront inhomogeneities present in this case introduced a large sampling uncertainty. Looking forward, there is a clear need for more observational campaigns focused on measuring near-surface turbulent fluxes in active regions of the atmosphere, such as in and around fronts in a translating frame of reference, and in situ aircraft observations provide a means to fulfill this need.

Acknowledgments. The authors thank the U.K.'s Facility for Airborne Atmospheric Measurements (FAAM), which undertook the flight discussed in this paper; Ian Renfrew, who led the T-NAWDEX Pilot campaign; and Doug Parker, who was the mission scientist on this flight and suggested the use of a closed circuit in a system-relative sense. The authors also thank Kirsty Hanley for her help in setting up the model simulations. BH was supported by NERC through Grant 
NE/I005196/1 (Diabatic Influences on Mesoscale Structures in Extratropical Storms).

\section{REFERENCES}

Apsley, M. L., K. J. Mulder, and D. M. Schultz, 2016: Reexamining the United Kingdom's greatest tornado outbreak: Forecasting the limited extent of tornadoes along a cold front. Wea. Forecasting, 31, 853-875, doi:10.1175/WAF-D-15-0131.1.

Best, M. J., and Coauthors, 2011: The Joint UK Land Environment Simulator (JULES), model description-Part 1: Energy and water fluxes. Geosci. Model Dev., 4, 677-699, doi:10.5194/ gmd-4-677-2011.

Boutle, I. A., J. E. J. Eyre, and A. P. Lock, 2014: Seamless stratocumulus simulation across the turbulent gray zone. Mon. Wea. Rev., 142, 1655-1668, doi:10.1175/MWR-D-13-00229.1.

Browning, K. A., and T. W. Harrold, 1970: Air motion and precipitation growth at a cold front. Quart. J. Roy. Meteor. Soc., 96, 369-389, doi:10.1002/qj.49709640903.

Catto, J. L., and S. Pfahl, 2013: The importance of fronts for extreme precipitation. J. Geophys. Res. Atmos., 118, 10 791-10 801, doi:10.1002/jgrd.50852.

Clark, M. R., and D. J. Parker, 2014: On the mesoscale structure of surface wind and pressure fields near tornadic and nontornadic cold fronts. Mon. Wea. Rev., 142, 3560-3585, doi:10.1175/MWR-D-13-00395.1.

Clark, P., N. Roberts, H. Lean, S. P. Ballard, and C. CharltonPerez, 2016: Convection-permitting models: A step-change in rainfall forecasting. Meteor. Appl., 23, 165-181, doi:10.1002/ met.1538.

Cook, P. A., and I. A. Renfrew, 2015: Aircraft-based observations of air-sea turbulent fluxes around the British Isles. Quart. J. Roy. Meteor. Soc., 141, 139-152, doi:10.1002/qj.2345.

Davies, T., M. J. P. Cullen, A. J. Malcolm, M. H. Mawson, A. Staniforth, A. A. White, and N. Wood, 2005: A new dynamical core for the Met Office's global and regional modelling of the atmosphere. Quart. J. Roy. Meteor. Soc., 131, 1759-1782, doi:10.1256/qj.04.101.

Eden, P., 2010: November 2009: Very mild and exceptionally wet. Weather, 65, i-iv, doi:10.1002/wea.545.

—- and S. Burt, 2010: Extreme rainfall in Cumbria, 18-20 November 2009. Weather, 65, 14, doi:10.1002/wea.551.

Edwards, J. M., and A. Slingo, 1996: Studies with a flexible new radiation code. I: Choosing a configuration for a large-scale model. Quart. J. Roy. Meteor. Soc., 122, 689-719, doi:10.1002/ qj. 49712253107.

FAAM, 2014: FAAM B488 T-NAWDEX flight, number 3: Airborne atmospheric measurements from core instrument suite on board the BAE-146 aircraft. NCAS British Atmospheric Data Centre, accessed 1 July 2015, http://catalogue.ceda.ac.uk/ uuid/cd4e60b21cd44c1aae7782b1bd7fa6d5.

Gregory, D., and P. R. Rowntree, 1990: A mass flux convection scheme with representation of cloud ensemble characteristics and stability-dependent closure. Mon. Wea. Rev., 118, 1483-1506, doi:10.1175/1520-0493(1990)118<1483: AMFCSW $>2.0 . C O ; 2$.

Halliwell, C., 2007: Subgrid turbulence scheme. Met Office Unified Model Documentation Paper 28, 18 pp.

Hanley, K. E., R. S. Plant, T. H. M. Stein, R. J. Hogan, J. C. Nicol, H. W. Lean, C. Halliwell, and P. A. Clark, 2015: Mixing-length controls on high-resolution simulations of convective storms. Quart. J. Roy. Meteor. Soc., 141, 272-284, doi:10.1002/qj.2356.
_ A. I. Barrett, and H. W. Lean, 2016: Simulating the 20 May 2013 Moore, Oklahoma tornado with a 100-metre grid-length NWP model. Atmos. Sci. Lett., 17, 453-461, doi:10.1002/ asl.678.

Harvey, B. J., 2011: Surface effects in quasi-geostrophic dynamics. Ph.D. thesis, University of Reading, 139 pp.

Hobbs, P. V., and K. R. Biswas, 1979: The cellular structure of narrow cold-frontal rainbands. Quart. J. Roy. Meteor. Soc., 105, 723-727, doi:10.1002/qj.49710544516.

_ , and P. O. G. Persson, 1982: The mesoscale and microscale structure and organization of clouds and precipitation in midlatitude cyclones. Part V: The substructure of narrow coldfrontal rainbands. J. Atmos. Sci., 39, 280-295, doi:10.1175/ 1520-0469(1982)039<0280:TMAMSA > 2.0.CO;2.

Hoskins, B. J., and F. P. Bretherton, 1972: Atmospheric frontogenesis models: Mathematical formulation and solution. J. Atmos. Sci., 29, 11-37, doi:10.1175/1520-0469(1972)029<0011: AFMMFA $>2.0 . \mathrm{CO} ; 2$.

James, P. K., and K. A. Browning, 1979: Mesoscale structure of line convection at surface cold fronts. Quart. J. Roy. Meteor. Soc., 105, 371-382, doi:10.1002/qj.49710544404.

Kawashima, M., 2011: Numerical study of horizontal shear instability waves along narrow cold frontal rainbands. J. Atmos. Sci., 68, 878-903, doi:10.1175/2010JAS3599.1.

Knippertz, P., J. M. Chagnon, A. Foster, L. Lathouwers, J. H. Marsham, J. Methven, and D. J. Parker, 2010: Research flight observations of a prefrontal gravity wave near the southwestern UK. Weather, 65, 293-297, doi:10.1002/ wea.632.

Lean, H. W., P. A. Clark, M. Dixon, N. M. Roberts, A. Fitch, R. Forbes, and C. Halliwell, 2008: Characteristics of highresolution versions of the Met Office Unified Model for forecasting convection over the United Kingdom. Mon. Wea. Rev., 136, 3408-3424, doi:10.1175/2008MWR2332.1.

Lock, A., A. R. Brown, M. R. Bush, G. M. Martin, and R. N. B. Smith, 2000: A new boundary layer mixing scheme. Part I: Scheme description and single-column model tests. Mon. Wea. Rev., 128, 3187-3199, doi:10.1175/1520-0493(2000)128<3187: ANBLMS $>2.0 . \mathrm{CO} ; 2$.

Mariotti, A., B. Legras, and D. Dritschel, 1994: Vortex stripping and the erosion of coherent structures in two-dimensional flows. Phys. Fluids, 6, 3954-3962, doi:10.1063/1.868385.

Martínez-Alvarado, O., H. Joos, J. Chagnon, M. Boettcher, S. L. Gray, R. S. Plant, J. Methven, and H. Wernli, 2014: The dichotomous structure of the warm conveyor belt. Quart. J. Roy. Meteor. Soc., 140, 1809-1824, doi:10.1002/qj.2276.

Mulder, K. J., and D. M. Schultz, 2015: Climatology, storm morphologies, and environments of tornadoes in the British Isles: 1980-2012. Mon. Wea. Rev., 143, 2224-2240, doi:10.1175/ MWR-D-14-00299.1.

Petersen, G. N., and I. A. Renfrew, 2009: Aircraft-based observations of air-sea fluxes over Denmark Strait and the Irminger Sea during high wind speed conditions. Quart. J. Roy. Meteor. Soc., 135, 2030-2045, doi:10.1002/qj.355.

Renfrew, I. A., and Coauthors, 2008: The Greenland Flow Distortion Experiment. Bull. Amer. Meteor. Soc., 89, 1307-1324, doi:10.1175/2008BAMS2508.1.

Shakespeare, C. J., and J. R. Taylor, 2014: The spontaneous generation of inertia-gravity waves during frontogenesis forced by large strain: Theory. J. Fluid Mech., 757, 817-853, doi:10.1017/jfm.2014.514.

Sinclair, V. A., and D. Keyser, 2015: Force balances and dynamical regimes of numerically simulated cold fronts within the 
boundary layer. Quart. J. Roy. Meteor. Soc., 141, 2148-2164, doi:10.1002/qj.2512.

Smart, D. J., and K. A. Browning, 2009: Morphology and evolution of cold-frontal misocyclones. Quart. J. Roy. Meteor. Soc., $\mathbf{1 3 5}$, 381-393, doi:10.1002/qj.399.

Stein, T. H. M., R. J. Hogan, P. A. Clark, C. E. Halliwell, K. E. Hanley, H. W. Lean, J. C. Nicol, and R. S. Plant, 2015: The DYMECS project: A statistical approach for the evaluation of convective storms in high-resolution NWP models. Bull. Amer. Meteor. Soc., 96, 939-951, doi:10.1175/BAMS-D-13-00279.1.

Vaughan, G., and Coauthors, 2015: Cloud banding and winds in intense European cyclones: Results from the DIAMET project. Bull. Amer. Meteor. Soc., 96, 249-265, doi:10.1175/ BAMS-D-13-00238.1.

Vosper, S., E. Carter, H. Lean, A. Lock, P. Clark, and S. Webster, 2013: High resolution modelling of valley cold pools. Atmos. Sci. Lett., 14, 193-199, doi:10.1002/asl2.439.

Williams, R. T., 1974: Numerical simulation of steady-state fronts. J. Atmos. Sci., 31, 1286-1296, doi:10.1175/ 1520-0469(1974)031<1286:NSOSSF $>2.0 . \mathrm{CO} ; 2$.

Wilson, D. R., and S. P. Ballard, 1999: A microphysically based precipitation scheme for the UK Meteorological Office Unified Model. Quart. J. Roy. Meteor. Soc., 125, 1607-1636, doi:10.1002/qj.49712555707. 